Pacific

Journal of

Mathematics

\title{
BAR-NATAN'S KHOVANOV HOMOLOGY FOR COLOURED LINKS
}

MARCo MaCKAAY AND PAUl TURner 


\title{
BAR-NATAN'S KHOVANOV HOMOLOGY FOR COLOURED LINKS
}

\author{
MARCO MACKAAY AND PAUl TURNER
}

\begin{abstract}
Using Bar-Natan's link homology we define a homology theory for framed links whose components are labelled by irreducible representations of the group $U_{q}\left(\mathfrak{s l}_{2}\right)$. We then compute this explicitly.
\end{abstract}

\section{Introduction}

Khovanov [2005] defined a link homology theory categorifying the coloured Jones polynomial. He constructed a cochain complex associated to an oriented framed link whose components are labelled by irreducible representations of $U_{q}\left(\mathfrak{s l}_{2}\right)$ with the property that the graded Euler characteristic of the homology of this complex is the coloured Jones polynomial. His key idea is to interpret the formula

$$
J_{n}(K)=\sum_{i=0}^{\lfloor n / 2\rfloor}(-1)^{i}\left(\begin{array}{c}
n-i \\
i
\end{array}\right) J\left(K^{n-2 i}\right),
$$

where $K^{j}$ is the $j$-cable of the knot $K$, as the Euler characteristic of a complex involving the link homology of the cablings $K^{n-2 i}$, for $i=0, \ldots,\lfloor n / 2\rfloor$.

Cabling a knot or link immediately introduces an unmanageable number of crossings from a computational point of view. For this reason, explicit computations in coloured Khovanov theory are scarce. It is interesting therefore to follow Khovanov's prescription for categorifying the coloured Jones polynomial but in doing so replacing his original theory with a simpler link homology theory. In this paper we do this using the theory constructed by Bar-Natan [2005]. We use the singly graded (filtered) theory, which is defined over $\mathbb{F}_{2}$, obtained by setting the variable $H$ in that paper to be 1 . This theory is somewhat like Lee's deformation of Khovanov homology and was computed explicitly in [Turner 2004] following

MSC2000: 57M25.

Keywords: Khovanov homology, coloured links, Bar-Natan theory.

Mackaay was supported by the Fundação para a Ciência e a Tecnologia through POCTI (Programa Operacional Ciência, Tecnologia, Inovação), cofinanced by the European Community fund FEDER. Turner was supported by the European Commission through a Marie Curie fellowship and thanks the Institut de Recherche Mathématique Avancée in Strasbourg for its hospitality. 
the techniques of [Lee 2005]. Our choice of Bar-Natan theory over Lee theory was simply to avoid issues of sign.

The resulting "coloured" invariants will not be particularly interesting per se (compare computations of Lee's theory or Bar-Natan theory where the homology only depends on the linking matrix of the link). However, as a testing ground for categorifying "coloured" invariants such a simple theory is invaluable. Also, as the remarkable paper [Rasmussen 2004] shows, important topological information can be extracted by considering the associated filtration of a simple theory (in his case Lee's theory).

In Section 2 we begin by recalling Khovanov's categorification of the coloured Jones polynomial and follow this by the definition and calculation of Bar-Natan theory. This categorification procedure can be carried out for any link homology and we proceed by using Bar-Natan theory in place of Khovanov's theory, leading to the definition of what we call coloured Bar-Natan theory. We calculate this explicitly for knots in Section 3, where the main results are Theorems 3.7 and 3.9. We extend this to links in Theorem 4.4 of Section 4. Finally we end with a short section on an alternative definition of the coloured theory suggested by Khovanov.

\section{Defining coloured Bar-Natan theory}

Khovanov's categorification of the coloured Jones polynomial. Let $L$ be an oriented framed link with each component labelled by an irreducible representation of $U_{q}\left(\mathfrak{s l}_{2}\right)$. Such representations are parametrized by $\mathbb{N}$, thus a coloured link is a link with each component labelled or coloured by a natural number. If this number is zero for a particular component we can simply delete this component, so we can assume throughout that $n \geq 1$. In this section we consider for the sake of simplicity an oriented framed knot $K$ with colour $n$. Let $D$ be a diagram for $K$ whose blackboard framing corresponds to the given framing of $K$.

A dot-row is a row of $n$ dots within which a number of consecutive dots are paired. A typical dot-row can be seen in the figure, where $n=9$.

Given a dot-row $s$, set $p(s)$ to number of pairs in $s$. Consider the oriented graph whose vertices are dot-rows and which has an arrow from $s$ to $s^{\prime}$ if and only if all pairs in $s$ are pairs in $s^{\prime}$ and $p\left(s^{\prime}\right)=p(s)+1$.

Now attach to the vertex $s$ of this graph the link $D_{s}:=D^{n-2 p(s)}$, where here $D^{k}$ means the $k$-cable of the diagram $D$. Each single dot in the dot-row corresponds to a cable-strand. Such a cable is oriented so that adjacent cable-strands have opposite orientations. To an edge $e$ with tail $s$ and head $s^{\prime}$ attach the cobordism $S_{e}$ which is the identity everywhere except at the two single dots in $s$ corresponding to the extra pair in $s^{\prime}$. For these two strands, the cobordism is the annulus with two inputs and 
no outputs, as illustrated here:

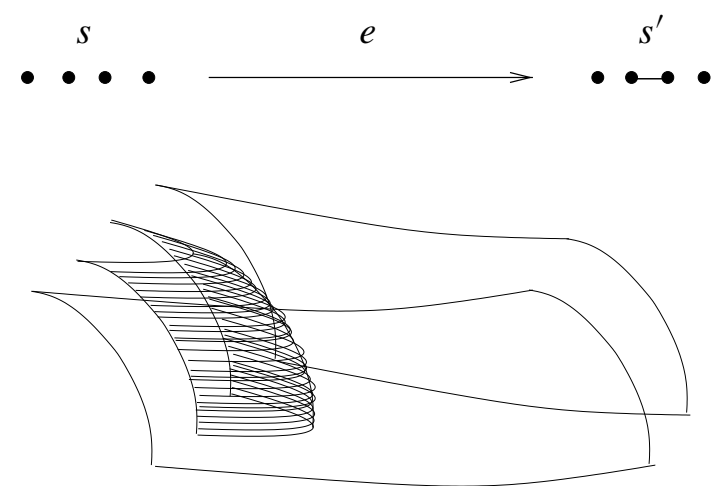

The cochain complex for the coloured theory is now obtained by applying Khovanov homology to this graph of links and link-cobordisms. More precisely, set

$$
C^{i}=\bigoplus_{s} \mathrm{KH}^{*, *}\left(D_{s} ; \mathbb{F}_{2}\right),
$$

where the sum is over vertices $s$ such that $p(s)=i$. The differential $d: C^{i} \rightarrow C^{i+1}$ is defined as follows. Given a dot-row $s$ and $v \in \mathrm{KH}^{* * *}\left(D_{s} ; \mathbb{F}_{2}\right)$, set

$$
d(v)=\sum\left(S_{e}\right)_{*}(v)
$$

where $\left(S_{e}\right)_{*}$ is the map in Khovanov homology induced by the cobordism $S_{e}$.

Coloured Khovanov theory is then defined as the homology of this complex, that is

$$
\mathrm{KH}_{n}^{i}\left(K ; \mathbb{F}_{2}\right)=H_{i}\left(C^{*}, d\right) .
$$

Each $C^{k}$ is bigraded via the bigrading in Khovanov homology and this induces a trigrading on $\mathrm{KH}_{n}^{*}\left(K ; \mathbb{F}_{2}\right)$. We write

$$
\mathrm{KH}_{n}^{*}\left(K ; \mathbb{F}_{2}\right)=\bigoplus \mathrm{KH}_{n}^{i, j, k}\left(K ; \mathbb{F}_{2}\right) .
$$

This defines a three variable polynomial

$$
K h_{n}(r, t, q)=\sum_{i, j, k} r^{i} t^{j} q^{k} \operatorname{dim}_{\mathbb{F}_{2}} \mathrm{KH}_{n}^{i, j, k}\left(K ; \mathbb{F}_{2}\right),
$$

from which one recovers the coloured Jones polynomial as

$$
J_{n}(K)=K h_{n}(-1,-1, q) .
$$

Khovanov [2005] has shown that all these objects are well defined and the definitions can be extended to coloured links, yielding an invariant of framed links. 
Moreover, $\mathrm{KH}^{*, *}\left(-; \mathbb{F}_{2}\right)$ can be replaced by any Khovanov theory, by which we mean a theory satisfying the axioms given in [Bar-Natan 2005] (see [Khovanov 2006] for a discussion of the universal theory). For theories over rings other than $\mathbb{F}_{2}$ signs must be introduced and care must be taken with the sign ambiguity introduced by the induced maps from cobordisms.

Bar-Natan Theory. This is a singly graded link homology theory defined using the (ungraded) Frobenius algebra $V=\mathbb{F}_{2}\{1, x\}$ with multiplication $\tilde{m}$ given by

$$
\tilde{m}(1,1)=1, \quad \tilde{m}(1, x)=x, \quad \tilde{m}(x, 1)=x, \quad \tilde{m}(x, x)=x ;
$$

comultiplication $\tilde{\Delta}$ by

$$
\tilde{\Delta}(1)=1 \otimes x+x \otimes 1+1 \otimes 1, \quad \tilde{\Delta}(x)=x \otimes x ;
$$

and unit and counit

$$
i(1)=1, \quad \epsilon(1)=0, \quad \epsilon(x)=1 .
$$

Denote the resulting homology of a link $L$ by $\mathrm{BN}^{*}(L)$. We will refer to this as BarNatan theory. In fact Bar-Natan defines a bigraded theory over $\mathbb{F}_{2}[H]$ with deg $H=$ -2 . The theory we are considering is the associated filtered theory obtained by setting $H=1$. It is isomorphic to what is called stable Bar-Natan theory in [Turner 2004]. This theory was computed in that paper, following the techniques developed in [Lee 2005].

Theorem 2.1. The dimension of $\mathrm{BN}^{*}(L)$ is $2^{k}$, where $k$ is the number of components in $L$.

In fact explicit generators can be found, as we now recall. There are $2^{k}$ possible orientations of the diagram for $L$. Given an orientation $\theta$ there is a canonical smoothing obtained by smoothing all positive crossings to 0 -smoothings and all negative crossings to 1-smoothings. For this smoothing one can divide the circles into two disjoint groups, Group 0 and Group 1 as follows. A circle belongs to Group 0 (Group 1) if it has the counterclockwise orientation and is separated from infinity by an even (odd) number of circles or if it has the clockwise orientation and is separated from infinity by an odd (even) number of circles. Now consider the element in the chain complex for $L$ defined by labelling each circle from Group 0 with $1+x$ and each circle from Group 1 with $x$, where we follow Bar-Natan's convention [2002] for the ordering of the circles. It can be shown that this defines a cycle which we denote $\mathfrak{s}_{\theta}$ and refer to as the canonical generator from the orientation $\theta$. To prove the above theorem in fact one shows

$$
\mathrm{BN}^{*}(L) \cong \mathbb{F}_{2}\left\{\left[\mathfrak{s}_{\theta}\right] \mid \theta \text { is an orientation of } L\right\} .
$$


Furthermore, it is possible to determine the degree of the generators in terms of linking numbers. Let $L_{1}, \ldots, L_{k}$ denote the components of $L$. Recalling that $L$ is oriented from the start, if we are given another orientation of $L$, say $\theta$, then we can obtain $\theta$ by starting with the original orientation and then reversing the orientation of a number of strands. Suppose that for the orientation $\theta$ the subset $E \subset\{1,2, \ldots, k\}$ indexes this set of strands to be reversed. Let $\bar{E}=\{1, \ldots, n\} \backslash E$. The degree of the corresponding generator $\left[\mathfrak{s}_{\theta}\right]$ is then given by

$$
\operatorname{deg}\left[\mathfrak{s}_{\theta}\right]=2 \sum_{l \in E, m \in \bar{E}} l k\left(L_{l}, L_{m}\right)
$$

where $l k\left(L_{l}, L_{m}\right)$ is the linking number (for the original orientation) between component $L_{l}$ and $L_{m}$.

Defining coloured Bar-Natan theory. Coloured Bar-Natan theory is the result of using $\mathrm{BN}^{*}(-)$ instead of $\mathrm{KH}^{*, *}\left(-; \mathbb{F}_{2}\right)$ in the definitions in the first part of this section. We now present the general definition for links.

Let $L$ be an oriented framed link with $k$ components and let $n_{j} \in \mathbb{N}$ be the colour of the $j$-th component. It will be convenient to assemble these into a vector $\underline{n}=\left(n_{1}, n_{2}, \ldots, n_{k}\right)$. Let $D$ be a diagram for $L$ whose blackboard framing agrees with the given framing of $L$ and let $D_{1}, D_{2}, \ldots, D_{k}$ be the components.

We will consider vectors of dot-rows $\underline{s}=\left(s_{1}, s_{2}, \ldots, s_{k}\right)$, where $s_{j}$ is a dot-row with $n_{j}$ dots. Let $\underline{p}(\underline{s})=\left(p\left(s_{1}\right), \ldots, p\left(s_{k}\right)\right)$ and define $|\underline{p}(\underline{s})|=p\left(s_{1}\right)+\cdots+p\left(s_{k}\right)$ where as before $\bar{p}\left(s_{i}\right)=$ number of pairs in $s_{i}$. Take the dot-row vectors $\underline{s}$ as the vertices of an oriented graph which has an arrow $\underline{s} \rightarrow \underline{s}^{\prime}$ if and only if all pairs in $\underline{s}$ are pairs in $\underline{s}^{\prime}$ and $\left|\underline{p}\left(\underline{s}^{\prime}\right)\right|=|\underline{p}(\underline{s})|+1$.

To a dot-row vector $\underline{s}$ we attach the cable $D_{\underline{s}}=D^{\underline{n}-2} \underline{p} \underline{(s)}$, where the notation means that we take the $\left(n_{j}-2 p\left(s_{j}\right)\right)$-cable of the $j$-th component. Cables are oriented so that adjacent strands have opposite orientation. To an arrow $e: \underline{s} \rightarrow \underline{s}^{\prime}$ we attach the cobordism $S_{e}$ as on page 430; that is, we take the cobordism which is the identity everywhere except at the two strands associated to the single dots in $\underline{s}$ corresponding to the extra pair in $\underline{s}^{\prime}$. For these two strands, the cobordism is the annulus with two inputs and no outputs.

To this we now apply Bar-Natan theory. Set

$$
\mathrm{C}_{\underline{n}}^{i}(D)=\bigoplus_{\underline{s}} \mathrm{BN}^{*}\left(D_{\underline{s}}\right),
$$

where the sum is over all $\underline{s}$ such that $|\underline{p}(\underline{s})|=i$. This is a complex under the differential $d: \mathrm{C}_{\underline{n}}^{i}(D) \rightarrow \mathrm{C}_{\underline{n}}^{i+1}(D)$ defined for $v \in \mathrm{BN}^{*}\left(D_{\underline{s}}\right)$ by

$$
d(v)=\sum\left(S_{e}\right)_{*}(v)
$$


where $\left(S_{e}\right)_{*}$ is the map in Bar-Natan theory induced by the cobordism $S_{e}$ and the sum is over all edges $e$ with tail $\underline{s}$.

Coloured Bar-Natan theory is then defined by

$$
\mathrm{BN}_{\underline{n}}^{i}(L)=H_{i}\left(\mathrm{C}_{\underline{n}}^{*}(D), d\right) .
$$

Each $\mathrm{C}_{\underline{n}}^{i}(D)$ is graded which induces a bigrading on coloured Bar-Natan theory and we write

$$
\mathrm{BN}_{\underline{n}}^{i}(L)=\bigoplus \mathrm{BN}_{\underline{n}}^{i, j}(L) .
$$

It is easy to check that $\mathrm{BN}_{\underline{1}}^{0, *}(L) \cong \mathrm{BN}^{*}(L)$, where $\underline{1}=(1,1, \ldots, 1)$.

\section{Calculating coloured Bar-Natan theory for knots}

Let $K$ be a framed, oriented knot $K$ with framing $f_{K} \in \mathbb{Z}$ and coloured by $n \in \mathbb{N}$. Suppose this is presented via a diagram $D$ with blackboard framing $f_{K}$. Take a cross-section of the $n$-cable $D^{n}$ such that the original orientation of $D$ is upward and number the strands of $D^{n}$ with the numbers $1, \ldots, n$ from left to right. Recall that the $n$-cable is oriented such that adjacent strands have opposite orientation and we insist that the strand labelled 1 has the same orientation as $D$.

Given any orientation $\theta$ of $D^{n}$, let $\theta(i)$ be the orientation of the $i$-th cable. The symmetric group on $n$ letters $\mathfrak{S}_{n}$ acts on the set of orientations for $K^{n}$ as follows. Given $\sigma \in \mathfrak{S}_{n}$ set

$$
\sigma \theta(i)=\theta(\sigma(i))
$$

This induces an action of $\mathfrak{S}_{n}$ on the set of canonical generators

$$
\sigma \mathfrak{s}_{\theta}=\mathfrak{s}_{\sigma(\theta)}
$$

and hence an action on $\mathrm{BN}^{*}\left(K^{n}\right)$. This action is independent of the diagram we have chosen for $K$, because it commutes with the Reidemeister moves. This follows from [Rasmussen 2004, Proposition 2.3], which can easily be seen to hold for Bar-Natan theory as well. In our case the proposition says that any Reidemeister move between two knot diagrams induces the linear isomorphism between the homologies of the two diagrams which maps any canonical generator to the canonical generator corresponding to the compatible orientation.

Denote the vector space of symmetric elements under this action by

$$
\left(\mathrm{BN}^{*}\left(K^{n}\right)\right)^{\mathfrak{S}_{n}}=\left\{\alpha \in \mathrm{BN}^{*}\left(K^{n}\right) \mid \sigma \alpha=\alpha \text { for all } \sigma \in \mathfrak{S}_{n}\right\} .
$$

It is clear that $\left(\mathrm{BN}^{*}\left(K^{n}\right)\right)^{\mathfrak{S}_{n}}$ has a basis consisting of the elements $\sum\left[\mathfrak{s}_{\theta}\right]$, where the sum is over a $\mathfrak{S}_{n}$-orbit of orientations $\theta$. It follows that $\left(\mathrm{BN}^{*}\left(K^{n}\right)\right)^{\mathfrak{S}_{n}}$ has dimension $n+1$. Notice that using the diagram $D$ each $\mathfrak{S}_{n}$-orbit is determined 
solely by the number of cable strands whose orientation agrees with the original (alternating) orientation of $D^{n}$.

In order to calculate the coloured Bar-Natan theory we will need the following lemma. Let $e$ be an edge of the graph of dot-rows connecting $s$ to $s^{\prime}$ and let $S_{e}$ be the associated cobordism as defined on page 433. Suppose that strands $l$ and $l+1$ are contracted. An orientation $\theta$ of $L_{s}$ induces an orientation $\theta^{\prime}$ on $L_{s^{\prime}}$, simply by removing strands $l$ and $l+1$.

\section{Lemma 3.1.}

$$
\left(S_{e}\right)_{*}\left(\left[\mathfrak{s}_{\theta}\right]\right)= \begin{cases}{\left[\mathfrak{s}_{\theta^{\prime}}\right]} & \text { if } l \text { and } l+1 \text { has opposite orientations, } \\ 0 & \text { if } l \text { and } l+1 \text { have the same orientation }\end{cases}
$$

Proof. This is essentially a variation mod 2 on a result by Rasmussen. The cobor$\operatorname{dism} S_{e}$ can be presented by a movie starting with a 1-handle move which fuses two strands into one, followed by a sequence of type two Reidemeister moves, and ending with the removal of a circle.

The first of these is given at the chain level by multiplication or comultiplication. If the orientations of the two strands agree, they belong to different circles and the cobordism induces a multiplication. Based on Rasmussen's analysis [2004, Lemma 2.4] we see that the canonical generator will label one of these strands with $1+x$ and the other with $x$; thus their fusion will produce zero as required. If the orientations are opposite there are two possible cases. In the first, the two strands belong to different circles and the cobordism induces a multiplication. The label will be the same on the two and fusion will give that same label to the single fused circle. In the second case the two strands belong to the same circle, with one label of course, and the cobordism induces a comultiplication which produces two circles with that same label. In both cases we see that a canonical generator is mapped to a canonical generator.

As already remarked, by arguments similar to those in the proof of [Rasmussen 2004, Proposition 2.3], it can be seen that Reidemeister two moves take a canonical generator to a canonical generator.

Finally the removal of a circle is given by the counit which takes the value 1 on both $1+x$ and $x$.

Corollary 3.2. Let $\sigma$ be the permutation switching strands $l$ and $l+1$. Then

$$
\left(S_{e}\right)_{*}\left(\left[\mathfrak{s}_{\theta}\right]\right)=\left(S_{e}\right)_{*}\left(\left[\mathfrak{s}_{\sigma \theta}\right]\right) .
$$

We now calculate the coloured Bar-Natan theory in degree zero for a coloured knot.

Proposition 3.3. Let $K$ be a framed, oriented knot with colouring $n \in \mathbb{N}$. Then

$$
\mathrm{BN}_{n}^{0}(K)=\left(\mathrm{BN}^{*}\left(K^{n}\right)\right)^{\mathfrak{S}_{n}} .
$$


Proof. We must show that $\operatorname{Ker} d_{0}=\left(\mathrm{BN}^{*}\left(D^{n}\right)\right)^{\mathfrak{S}_{n}}$, where $d_{0}: C_{n}^{0}(D) \rightarrow C_{n}^{1}(D)$ is the differential. Notice that $d_{0}$ is the sum of $n-1$ contractions. Let $d_{0, l}$ be the induced map in Bar-Natan theory corresponding to the cobordism contracting strands $l$ and $l+1$. An element $v$ is in the kernel of $d_{0}$ if and only if it is the kernel of $d_{0, l}$ for $l=1, \ldots, n-1$.

Now let $v=\sum\left[\mathfrak{s}_{\theta}\right] \in\left(\mathrm{BN}^{*}\left(K^{n}\right)\right)^{\mathfrak{S}_{n}}$. We claim that $d_{0, l}(v)=0$. Firstly note that we can split $v$ into a sum over orientations where strands $l$ and $l+1$ are oriented the same way and orientations where they are oriented differently:

$$
v=\sum_{\text {same }}\left[\mathfrak{s}_{\theta}\right]+\sum_{\text {diff }}\left[\mathfrak{s}_{\theta}\right]
$$

Letting $\sigma$ be the transposition switching $l$ and $l+1$ the sum on the right can be replaced by a sum of elements $\left[\mathfrak{s}_{\theta}\right]+\left[\sigma \mathfrak{s}_{\theta}\right]$, where the sum is over orientations for which strand $l$ and $l+1$ are oriented as for the original orientation of $K^{n}$ :

$$
v=\sum_{\text {same }}\left[\mathfrak{s}_{\theta}\right]+\sum_{\text {orig }}\left(\left[\mathfrak{s}_{\theta}\right]+\left[\sigma \mathfrak{s}_{\theta}\right]\right)
$$

Thus

$$
\begin{array}{rlrl}
d_{0, l}(v) & =d_{0, l}\left(\sum_{\text {same }}\left[\mathfrak{s}_{\theta}\right]+\sum_{\text {orig }}\left(\left[\mathfrak{s}_{\theta}\right]+\left[\sigma \mathfrak{s}_{\theta}\right]\right)\right) \\
& =\sum_{\text {same }} d_{0, l}\left(\left[\mathfrak{s}_{\theta}\right]\right)+\sum_{\text {orig }}\left(d_{0, l}\left(\left[\mathfrak{s}_{\theta}\right]\right)+d_{0, l}\left(\left[\sigma \mathfrak{s}_{\theta}\right]\right)\right) \\
& =\sum_{\text {orig }} d_{0, l}\left(\left[\mathfrak{s}_{\theta}\right]\right)+d_{0, l}\left(\left[\sigma \mathfrak{s}_{\theta}\right]\right) & \text { (by Lemma 3.1) } \\
& =0 \bmod 2 \quad \quad \text { (by Corollary 3.2) }
\end{array}
$$

Since this is true for $l=1, \ldots, n-1$, we have shown $d_{0}(v)$ vanishes and hence that $\left(\mathrm{BN}^{*}\left(K^{n}\right)\right)^{\mathfrak{S}_{n}} \subseteq \operatorname{Ker} d_{0}$.

Now suppose that $v=\sum \lambda_{\theta}\left[\mathfrak{s}_{\theta}\right]$ is in the kernel of $d_{0}$ and let $l \in\{1, \ldots, n-1\}$. As above let $\sigma$ be the transposition switching strands $l$ and $l+1$. Suppose for an orientation $\theta$ we have $\lambda_{\theta} \neq 0$. If strands $l$ and $l+1$ have the same orientation in $\theta$ then $\sigma\left[\mathfrak{s}_{\theta}\right]=\left[\mathfrak{s}_{\theta}\right]$. If strands $l$ and $l+1$ have different orientations, then using the fact that $v \in \operatorname{Ker} d_{0, l}$, Lemma 3.1 implies that we must also have $\lambda_{\sigma \theta} \neq 0$. This gives us that $\sigma v=v$. This is true for all transpositions $\sigma=(l, l+1)$ for $l=1, \ldots, n-1$ and since $\mathfrak{S}_{n}$ in generated by such transpositions this shows that $\tau v=v$ for all $\tau \in \mathfrak{S}_{n}$. We conclude that $v$ lies in $\left(B^{*}\left(K^{n}\right)\right)^{\mathfrak{S}_{n}}$ and hence that $\operatorname{Ker} d_{0} \subseteq\left(\mathrm{BN}^{*}\left(K^{n}\right)\right)^{\mathfrak{S}_{n}}$, which finishes the proof.

To calculate the coloured Bar-Natan theory in higher degrees we need to first introduce a new family of complexes. For $m=0,1, \ldots, n$ define $C_{n}^{*}(m)$ as follows, 
where we suppress the diagram $D$ from the notation. Consider the graph of dotrows with $m$ dots. Now attach to the vertex $s$ of this graph the cable $D_{s}:=D^{n-2 p(s)}$. The first $(m-2 p(s))$-strands of this cable are associated with the $m-2 p(s)$ dots. The remaining $n-m$ cable strands are not associated to any dots. To edges we associate annulus cobordisms as before insisting that cobordisms are the identity on the last $n-m$ "free" cables. Now set

$$
C_{n}^{i}(m)=\bigoplus_{s} \mathrm{BN}^{*}\left(D_{s}\right)
$$

where the sum is over dot-rows $s$ such that $p(s)=i$. As above the arrows in the dot-row graph give rise to a differential. Let $H_{n}^{*}(m)$ denote the homology of this complex.

Notice that $C_{n-2}^{*}(m-2)[-1]$, where [-1] means a downward shift of one degree, can be identified with the subcomplex of $C_{n}^{*}(m)$ consisting of dot-rows with the last two dots always forming a pair. The quotient complex is seen to be $C_{n}^{*}(m-1)$, so there are short exact sequences

$$
0 \longrightarrow C_{n-2}^{*}(m-2)[-1] \longrightarrow C_{n}^{*}(m) \longrightarrow C_{n}^{*}(m-1) \longrightarrow 0
$$

This is illustrated here for $n=7$ and $m=4$ :
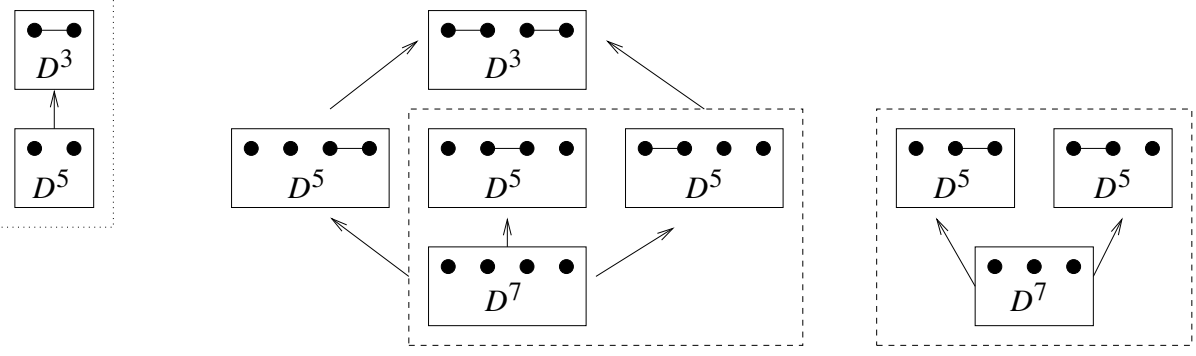

$C_{5}^{*}(2)[-1]$ $>C_{7}^{*}(4)$ $>C_{7}^{*}(3)$

Such a short exact sequence gives rise to a long exact sequence

$$
\cdots \longrightarrow H_{n-2}^{i-1}(m-2) \longrightarrow H_{n}^{i}(m) \longrightarrow H_{n}^{i}(m-1) \stackrel{\delta}{\longrightarrow} H_{n-2}^{i}(m-2) \longrightarrow \cdots
$$

In the next lemma we gather some useful results about the homology of these complexes. In part (3) we are using the fact that $\mathfrak{S}_{m}$ includes in $\mathfrak{S}_{n}$ as permutations of the first $m$ members of $\{1, \ldots, n\}$ and so $\mathfrak{S}_{m}$ acts on $\mathrm{BN}^{*}\left(K^{n}\right)$. 


\section{Lemma 3.4.}

$$
\begin{aligned}
& H_{n}^{*}(n) \cong \mathrm{BN}_{n}^{*}(K) . \\
& H_{n}^{i}(0) \cong H_{n}^{i}(1) \cong \begin{cases}\mathrm{BN}^{*}\left(K^{n}\right) & \text { if } i=0, \\
0 & \text { otherwise. }\end{cases} \\
& H_{n}^{0}(m) \cong\left(\mathrm{BN}^{*}\left(K^{n}\right)\right)^{\mathfrak{S}_{m}} . \\
& \operatorname{dim} H_{n}^{0}(m)=2^{n-m}(m+1) .
\end{aligned}
$$

Proof. Examining the definition above shows that $C_{n}^{*}(n)$ is the complex defining coloured Bar-Natan theory and also that

$$
C_{n}^{i}(0) \cong C_{n}^{i}(1) \cong \begin{cases}\mathrm{BN}^{*}\left(K^{n}\right) & \text { if } i=0, \\ 0 & \text { otherwise, }\end{cases}
$$

from which (1) and (2) follow immediately. Part (3) is essentially a corollary to Proposition 3.3 and part (4) follows from part (3).

Proposition 3.5. $H_{n}^{1}(m)=0$ for all $n \in \mathbb{N}$ and $m=0,1, \ldots, n$.

Proof. The proof is by induction on $m$. By Lemma 3.4(2) we have $H_{n}^{1}(0)=$ $H_{n}^{1}(1)=0$ for all $n$ which starts the induction. Now suppose that $H_{n}^{1}(m)=0$ for $m=0,1, \ldots, M$ and $n \geq M$. We claim $H_{n}^{1}(M+1)=0$ for $n \geq M+1$. There is a piece of long exact sequence as follows.

$$
0 \longrightarrow H_{n}^{0}(M+1) \longrightarrow H_{n}^{0}(M) \longrightarrow H_{n-2}^{0}(M-1) \longrightarrow H_{n}^{1}(M+1) \longrightarrow H_{n}^{1}(M)
$$

The group on the right is trivial by the inductive hypothesis. Using Lemma 3.4(4) we see that the first three groups in the sequence have dimensions $2^{n-M-1}(M+2)$, $2^{n-M}(M+1)$ and $2^{n-M-1} M$ respectively. Since the alternating sum of dimensions in an exact sequence is zero this gives

$$
\begin{aligned}
\operatorname{dim} H_{n}^{1}(M+1) & =2^{n-M-1}(M+2)-2^{n-M}(M+1)+2^{n-M-1} M \\
& =2^{n-M-1}(2 M+2)-2^{n-M}(M+1)=0 .
\end{aligned}
$$

Hence $H_{n}^{1}(M+1)=0$ as required.

Proposition 3.6. $H_{n}^{i}(m)=0$ for all $i \geq 1$ and for all $n \in \mathbb{N}$ and $m=0,1, \ldots, n$.

Proof. The proof is by induction on $i$. Proposition 3.5 starts the induction with $i=1$. Now suppose the result holds for $1 \leq i<j$. We claim that the result holds for $i=j$, i.e., $H_{n}^{j}(m)=0$ for all $n$ and $m=0,1, \ldots, n$. To show this we do a second induction, this time on $m$, where the base case is again provided by Lemma $3.4(2)$. Now suppose $H_{n}^{j}(m)=0$ for $m=0,1, \ldots, M$. Then we have a piece of long exact sequence

$$
H_{n-2}^{j-1}(M-1) \longrightarrow H_{n}^{j}(M+1) \longrightarrow H_{n}^{j}(M) .
$$


The group on the right is trivial by the induction on $m$ and the group on the left is trivial by the induction on $i$, hence the middle group is trivial too.

In particular when $m=n$ we have $\operatorname{BN}_{n}^{i}(K)=H_{n}^{i}(n)=0$ for $i \geq 1$. Combining this with Proposition 3.3 we have:

\section{Theorem 3.7.}

$$
\mathrm{BN}_{n}^{i}(K)= \begin{cases}\left(\mathrm{BN}^{*}\left(K^{n}\right)\right)^{\mathfrak{S}_{n}} & \text { if } i=0, \\ 0 & \text { otherwise. }\end{cases}
$$

Theorem 3.7 is similar to [Khovanov 2005, Theorem 1]. Our proof, although similar in spirit, is more complicated because $\mathrm{BN}^{*}\left(K^{n}\right) \varsubsetneqq \mathrm{BN}^{*}(K)^{\otimes n}$.

\section{Corollary 3.8.}

$$
\operatorname{dim} \mathrm{BN}_{n}^{*}(K)=n+1 .
$$

Recall that in each degree there is a second grading. For a complete calculation it remains for us to describe this for the zeroth homology group. Note that if $\mathfrak{s}_{\theta} \in \mathrm{BN}^{i}\left(K^{n}\right)$ then for $\sigma \in \mathfrak{S}_{n}$ we have $\mathfrak{s}_{\sigma \theta} \in \mathrm{BN}^{i}\left(K^{n}\right)$, because the number of negative crossings in $K^{n}$ is the same for both orientations, which shows us that the grading on $\mathrm{BN}^{*}\left(K^{n}\right)$ induces a well defined internal grading on $\mathrm{BN}_{n}^{0}(K)$. We write $\mathrm{BN}_{n}^{0}(K)=\bigoplus \mathrm{BN}_{n}^{0, i}(K)$.

Theorem 3.9. If $n$ is even then

$$
\mathrm{BN}_{n}^{0, i}(K)= \begin{cases}\mathbb{F}_{2} & \text { if } i=0, \\ \mathbb{F}_{2} \oplus \mathbb{F}_{2} & \text { if } i=-2 k^{2} f_{K}, \text { for } k=1, \ldots, \frac{1}{2} n, \\ 0 & \text { otherwise. }\end{cases}
$$

If $n$ is odd then

$$
\mathrm{BN}_{n}^{0, i}(K)= \begin{cases}\mathbb{F}_{2} \oplus \mathbb{F}_{2} & \text { if } i=-2 k(k+1) f_{K} \text { for } k=0, \ldots, \frac{1}{2}(n-1), \\ 0 & \text { otherwise. }\end{cases}
$$

Proof. Suppose that $n$ is even. In this case the orientation given to the $n$-cable of $K$ has $\frac{n}{2}$ strands agreeing with the given orientation of $K$ and $n / 2$ strands with the opposite orientation. Recall that an $\mathfrak{S}_{n}$-orbit is determined by the number of strands agreeing with the original orientation. Moreover, Corollary 3.2 implies that any element in a given orbit has the same internal degree. Note that $S_{e}$ preserves the internal degrees. If the orbit of $\theta$ has more than one element, then there exists a transposition $\sigma$ such that $\theta$ and $\sigma \theta$ are different. By the same corollary we know that $\mathfrak{s}_{\theta}$ and $\mathfrak{s}_{\sigma \theta}$ are mapped to the same nonzero element, so they have to have the same degree.

The given orientation of $K^{n}$ has $n / 2$ strands agreeing with $K$ and using the description above in the section on Bar-Natan theory (page 432) it is immediate 
that the canonical generator in $\mathrm{BN}^{*}\left(K^{n}\right)$ corresponding to this orientation has degree zero (the set $E$ in the theorem is the empty set). Thus the class in $\mathrm{BN}_{n}^{0}(K)$ determined by the orbit of this has internal grading zero.

Let $D_{1}, \ldots, D_{n}$ be the strands of the $n$-cable diagram and note that $l k\left(D_{2 i}, D_{l}\right)=$ $l k\left(D_{2}, D_{l}\right)$ and moreover that this is equal to $f_{K}$ if $l$ is even and $-f_{K}$ if $l$ is odd.

Now let $E=\{2,4, \ldots, 2 k\}$ for $k=1,2, \ldots, n / 2$ and consider the orientation of $K^{n}$ obtained by reversing the orientation of strands numbered by elements of $E$. Such an orientation has $n / 2+k$ strands agreeing with the orientation of $K$. We see that the degree of the canonical generator defined by this orientation is given by

$$
\begin{aligned}
2 \sum_{j \in E, l \in \bar{E}} l k\left(D_{j}, D_{l}\right) & =2\left(\sum_{l \in \bar{E}} l k\left(D_{2}, D_{l}\right)+\cdots+l k\left(D_{2 k}, D_{l}\right)\right)=2 k \sum_{l \in \bar{E}} l k\left(D_{2}, D_{l}\right) \\
& =2 k\left(\sum_{l \in\{2 k+2, \ldots, n\}} l k\left(D_{2}, D_{l}\right)+\sum_{l \text { odd }} l k\left(D_{2}, D_{l}\right)\right) \\
& =2 k\left(\sum_{l \in\{2 k+2, \ldots, n\}} f_{K}+\sum_{l \text { odd }}\left(-f_{K}\right)\right)=-2 k^{2} f_{K} .
\end{aligned}
$$

Thus the $\mathfrak{S}_{n}$-orbit containing this orientation has grading $-2 k^{2} f_{K}$. This accounts for the $\mathfrak{S}_{n}$-orbits with more than $n / 2$ strands agreeing with $K$.

To account for the orbits with less than $n / 2$ strands agreeing with $K$, let

$$
E=\{1,3, \ldots, 2 k-1\}
$$

for $k=1,2, \ldots, n / 2$ and consider the orientation of $K^{n}$ obtained by reversing the orientation of strands in $E$. This orientation has $n / 2-k$ strands agreeing with $K$. By a similar computation to the above we see that the degree of the canonical generator defined by this orientation is given by $-2 k^{2} f_{K}$ thus, combining with the above, giving a total of two generators in this degree.

This finishes the proof for $n$ even.

For $n$ odd the argument is similar with the difference that the given orientation of $K^{n}$ and the orientation from $E=\{1\}$ both give classes in degree zero. The orientations from $E=\{2,4, \ldots, 2 k\}$ and $E=\{1,3, \ldots, 2 k+1\}$ for $k=1,2, \ldots$, $\frac{1}{2}(n-1)$ both give rise to classes in degree $-2 k(k+1) f_{K}$. A slight rearrangement of indexing gives the statement of the theorem for $n$ odd.

Recall that each generator $\alpha$ is of the form $\sum_{\theta \in X}\left[\mathfrak{s}_{\theta}\right]$, where $X$ is a $\mathfrak{S}_{n}$-orbit of orientations. It is easy to see that $\bar{\alpha}:=\sum_{\theta \in X}\left[\mathfrak{s}_{\bar{\theta}}\right]$ is also a generator, because $\sigma \bar{\theta}=\overline{\sigma \theta}$, for any $\sigma \in \mathfrak{S}_{n}$. Moreover, by inspecting the above proof one can see that both $\alpha$ and $\bar{\alpha}$ have the same grading. Thus for each $\mathbb{F}_{2} \oplus \mathbb{F}_{2}$ in Theorem 3.9 if 
$\alpha$ generates one copy of $\mathbb{F}_{2}$ then $\bar{\alpha}$ generates the other. Note that if $n$ is even and $\alpha$ is the generator of degree 0 , then $\bar{\alpha}=\alpha$.

\section{Calculating coloured Bar-Natan theory for links}

Let $L$ be an oriented, framed link with $k$ components and let $n_{j} \in \mathbb{N}$ be the colouring of the $j$-th component. Let $D$ be a diagram for $L$ whose blackboard framing is the given framing of $L$.

As above for the case of knots we need a new family of complexes in order to calculate the coloured Bar-Natan theory of $L$. For each $j=1, \ldots, k$ choose $m_{j} \in \mathbb{N}$ and set $\underline{m}=\left(m_{1}, \ldots, m_{k}\right)$. We say that $\underline{m}$ is allowable if $1 \leq m_{j} \leq n_{j}$ for $j=1, \ldots, k$. We define the length of $\underline{m}$ by $|\underline{m}|=m_{1}+\cdots+m_{k}$.

Now consider the oriented graph whose vertices are vectors of dot-rows $\underline{s}=$ $\left(s_{1}, \ldots, s_{k}\right)$, where $s_{j}$ is a dot-row of $m_{j}$ dots. Arrows in the graph are defined using the same construction as on page 433 coordinatewise. Now attach $D_{\underline{s}}=D^{\underline{n}-2 \underline{p}(\underline{s})}$ to the vertex $\underline{s}$, where recall that $\underline{p}(\underline{s})=\left(p\left(s_{1}\right), \ldots, p\left(s_{k}\right)\right)$. For the $j$-th component, the first $m_{j}-2 p\left(s_{j}\right)$ strands of $D^{n_{j}-2 p\left(s_{j}\right)}$ are identified with the $m_{j}-2 p\left(s_{j}\right)$ dots, and the remaining $n_{j}-m_{j}$ strands are "free". As usual attach cobordisms to edges. Now set

$$
C_{\underline{n}}^{i}(\underline{m})=\bigoplus_{\underline{s}} \mathrm{BN}^{*}\left(D_{\underline{s}}\right),
$$

where the sum is over vectors of dot-rows $\underline{s}$ such that $|\underline{p}(\underline{s})|=p\left(s_{1}\right)+\cdots+p\left(s_{k}\right)=$ $i$. As above the arrows in the graph give rise to a differential. Let $H_{\underline{n}}^{*}(\underline{m})$ denote the homology of this complex.

Let $\underline{e}_{i}=(0, \ldots, 0,1,0, \ldots, 0)$ with a 1 in the $i$-th place. Since the arrows in the graph are defined coordinatewise, there are short exact sequences

$$
0 \longrightarrow C_{\underline{n}-2 \underline{e}_{i}}^{*}\left(\underline{m}-2 \underline{e}_{i}\right)[-1] \longrightarrow C_{\underline{n}}^{*}(\underline{m}) \longrightarrow C_{\underline{n}}^{*}\left(\underline{m}-\underline{e}_{i}\right) \longrightarrow 0
$$

giving rise to long exact sequences

$$
\cdots \longrightarrow H_{\underline{n}-2 \underline{e}_{i}}^{i-1}\left(\underline{m}-2 \underline{e}_{i}\right) \rightarrow H_{\underline{n}}^{i}(\underline{m}) \rightarrow H_{\underline{n}}^{i}\left(\underline{m}-\underline{e}_{i}\right) \stackrel{\delta}{\longrightarrow} H_{\underline{n}-2 \underline{e}_{i}}^{i}\left(\underline{m}-2 \underline{e}_{i}\right) \longrightarrow \cdots
$$

The symmetric group $\mathfrak{S}_{n_{j}}$ acts on the orientations of the $n_{j}$-cable of the $j$-th component and so $\mathfrak{S}_{\underline{n}}=\mathfrak{S}_{n_{1}} \times \cdots \times \mathfrak{S}_{n_{k}}$ acts on the set of orientations of $L^{\underline{n}}$. As before this induces an action on $\mathrm{BN}^{*}\left(L^{\underline{n}}\right)$. Letting

$$
\mathfrak{S}_{\underline{m}}=\mathfrak{S}_{m_{1}} \times \cdots \times \mathfrak{S}_{m_{k}} \subset \mathfrak{S}_{n_{1}} \times \cdots \times \mathfrak{S}_{n_{k}}=\mathfrak{S}_{\underline{n}},
$$

we see that $\mathfrak{S}_{\underline{m}}$ acts on $\mathrm{BN}^{*}\left(L^{\underline{n}}\right)$ too. We will write $\left(\mathrm{BN}^{*}\left(L^{\underline{n}}\right)\right)^{\mathfrak{S}_{\underline{m}}}$ to denote the space of symmetric elements under this action.

There is an analogue of Lemma 3.4 for links.

Lemma 4.1. (1) $H_{\underline{n}}^{*}(\underline{n}) \cong \mathrm{BN}_{\underline{n}}^{*}(L)$. 
(2) If $\underline{m}$ is such that $0 \leq m_{j} \leq 1$ for $j=1, \ldots, k$ then

$$
H_{\underline{n}}^{i}(\underline{m}) \cong \begin{cases}\mathrm{BN}^{*}\left(L^{\underline{n}}\right) & \text { if } i=0, \\ 0 & \text { otherwise. }\end{cases}
$$

(3) If $\underline{m}$ is allowable then

$$
\begin{gathered}
H_{\underline{n}}^{0}(\underline{m}) \cong\left(\mathrm{BN}^{*}\left(L^{\underline{n}}\right)\right)^{\mathfrak{S}_{\underline{m}}} . \\
\operatorname{dim} H_{\underline{n}}^{0}(\underline{m})=\prod_{j=1}^{k} 2^{n_{j}-m_{j}}\left(m_{j}+1\right) .
\end{gathered}
$$

Proof. The only nontrivial thing to check is part (3), which is an easy generalisation of Proposition 3.3.

The next results are the analogues of Propositions 3.5 and 3.6 and Theorem 3.7.

Proposition 4.2. $H_{\underline{n}}^{1}(\underline{m})=0$ for all $\underline{n}$ and all allowable $\underline{m}$.

Proof. The proof is by induction on the length of $\underline{m}$. The induction is started by Lemma 4.1(2). Suppose that $H_{n}^{1}(\underline{m})=0$ for all allowable $\underline{m}$ with $|\underline{m}|<M$. Now let $\underline{m}$ be such that $|\underline{m}|=M$. Using Lemma 4.1(2) we can suppose that for some $i$ we have $m_{i} \geq 2$. There is a piece of long exact sequence

$$
0 \longrightarrow H_{\underline{n}}^{0}(\underline{m}) \rightarrow H_{\underline{n}}^{0}\left(\underline{m}-\underline{e}_{i}\right) \rightarrow H_{\underline{n}-2 \underline{e}_{i}}^{0}\left(\underline{m}-2 \underline{e}_{i}\right) \rightarrow H_{\underline{n}}^{1}(\underline{m}) \rightarrow H_{\underline{n}}^{1}\left(\underline{m}-\underline{e}_{i}\right) .
$$

Since $\left|\underline{m}-\underline{e}_{i}\right|=M-1$ the group on the right is zero by the inductive hypothesis. Using Lemma 4.1(4) the first three groups have dimensions as follows.

$$
\begin{gathered}
\operatorname{dim} H_{\underline{n}}^{0}(\underline{m})=\prod_{j} 2^{n_{j}-m_{j}}\left(m_{j}+1\right) \\
\operatorname{dim} H_{\underline{n}}^{0}\left(\underline{m}-\underline{e}_{i}\right)=2^{n_{i}-m_{i}+1} m_{i} \prod_{j \neq i} 2^{n_{j}-m_{j}}\left(m_{j}+1\right) \\
\operatorname{dim} H_{\underline{n}-2 \underline{e}_{i}}^{0}\left(\underline{m}-2 \underline{e}_{i}\right)=2^{n_{i}-m_{i}}\left(m_{i}-1\right) \prod_{j \neq i} 2^{n_{j}-m_{j}}\left(m_{j}+1\right)
\end{gathered}
$$

Using the fact that the alternating sum of dimensions in an exact sequence is zero we get

$$
\begin{aligned}
\operatorname{dim} H_{\underline{n}}^{1}(\underline{m}) & =\left(2^{n_{i}-m_{i}}\left(m_{i}+1\right)-2^{n_{i}-m_{i}+1} m_{i}+2^{n_{i}-m_{i}}\left(m_{i}-1\right)\right) \prod_{j \neq i} 2^{n_{j}-m_{j}}\left(m_{j}+1\right) \\
& =2^{n_{i}-m_{i}}\left(m_{i}+1-2 m_{i}+m_{i}-1\right) \prod_{j \neq i} 2^{n_{j}-m_{j}}\left(m_{j}+1\right)=0 .
\end{aligned}
$$

This shows $H_{\underline{n}}^{1}(\underline{m})=0$ as required.

Proposition 4.3. $H_{\underline{n}}^{i}(\underline{m})=0$ for all $i \geq 1$ and for all $\underline{n}$ and allowable $\underline{m}$. 
Proof. We use induction on $i$ which is started by Proposition 4.2. Suppose $H_{n}^{i}(\underline{m})=$ 0 for $1 \leq i<j$. We now claim that $H_{\underline{n}}^{j}(\underline{m})=0$. To show this we use a second induction this time on the length of $\underline{m}$ which is started by Lemma 4.1(2). Suppose $H_{\underline{n}}^{j}(\underline{m})=0$ for $|\underline{m}|<M$. We claim that for $\underline{m}$ satisfying $|\underline{m}|=M$ we have $H_{\underline{n}}^{j}(\underline{m})=0$. There is a piece of long exact sequence

$$
H_{\underline{n}-2 \underline{e}_{i}}^{j-1}\left(\underline{m}-2 \underline{e}_{i}\right) \rightarrow H_{\underline{n}}^{j}(\underline{m}) \longrightarrow H_{\underline{n}}^{j}\left(\underline{m}-\underline{e}_{i}\right) .
$$

The group on the right is zero by the second induction since $\left|\underline{m}-\underline{e}_{i}\right|<M$ and the group on the left is zero by the first induction, thus the middle group is zero too.

\section{Theorem 4.4.}

$$
\mathrm{BN}_{\underline{n}}^{i}(L)= \begin{cases}\left(\mathrm{BN}^{*}\left(L^{\underline{n}}\right)\right)^{\mathfrak{S}_{\underline{n}}} & \text { if } i=0, \\ 0 & \text { otherwise. }\end{cases}
$$

Proof. From parts (1) and (3) of Lemma 4.1 we observe that $\mathrm{BN}_{\underline{n}}^{0}(L) \cong H_{\underline{n}}^{0}(\underline{n}) \cong$ $\left(\mathrm{BN}^{*}\left(L^{\underline{n}}\right)\right)^{\mathfrak{S}_{\underline{n}}}$. Proposition 4.3 shows that $\mathrm{BN}_{\underline{n}}^{i}(L) \cong H_{\underline{n}}^{i}(\underline{n})=0$, for all $i \geq 1$.

\section{Corollary 4.5.}

$$
\operatorname{dim} \mathrm{BN}_{\underline{n}}^{*}(L)=\prod_{j=1}^{k}\left(n_{j}+1\right) .
$$

We now consider the generators of $\mathrm{BN}_{n}^{*}(L)$. For each $i$ let $E_{i}$ be a subset of $\left\{1, \ldots, n_{i}\right\}$ and define $\bar{E}_{i}=\left\{1, \ldots, n_{i}\right\} \backslash E_{i}$. We denote the collection $\left\{E_{i}\right\}_{i=1, \ldots, k}$ by $\underline{E}$. Given $\underline{E}$ define

$\lambda_{i, l}=\#\left\{(j, m) \in E_{i} \times \bar{E}_{l} \mid j\right.$ and $m$ have the same parity $\}$

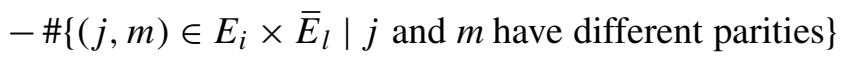

$$
=\sum_{j \in E_{i}} \sum_{m \in \bar{E}_{l}}(-1)^{j+m} .
$$

The collection $\underline{E}$ is said to be admissible if each $E_{i}$ is either empty or of one of the following forms:

$$
\begin{aligned}
& E_{i}=\{2,4, \ldots, 2 p\} \quad \text { for some } p \in\left\{1, \ldots,\left\lfloor n_{i} / 2\right\rfloor\right\}, \\
& E_{i}=\{1,3, \ldots, 2 p-1\} \text { for some } p \in\left\{1, \ldots,\left\lceil n_{i} / 2\right\rceil\right\} .
\end{aligned}
$$

Each admissible $\underline{E}$ determines an orientation of $L^{\underline{n}}$ by reversing the orientation of the strands indexed by $E_{i}$ in the $n_{i}$-cable of the $i$-th component. As explained earlier, this defines a cocycle $s_{\underline{E}}$ and hence a class $\left[s_{\underline{E}}\right] \in \mathrm{BN}^{*}\left(L^{\underline{n}}\right)$. The sum of elements in the $\mathfrak{S}_{\underline{n}}$-orbit of $\left[s_{\underline{E}}\right]$ is a generator of $\operatorname{BN}_{n}^{0}(L) \cong\left(\operatorname{BN}^{*}\left(L^{\underline{n}}\right)\right)^{\mathfrak{S}_{\underline{n}}}$. All generators arise in this way and there is a one-to-one correspondence between the admissible $\underline{E}$ and generators of $\operatorname{BN}_{\underline{n}}^{0}(L)$.

Recall that $\mathrm{BN}_{\underline{n}}^{*}(L)$ is in fact bigraded, the second grading being inherited from the grading on Bar-Natan theory. Here is the analogue of Theorem 3.9 for knots: 
Theorem 4.6. Let $\underline{E}$ be an admissible collection. The generator of $\mathrm{BN}_{n}^{0, *}(L)$ corresponding to $\underline{E}$ has grading

$$
2 \sum_{i=1}^{k} \sum_{l=1}^{k} \lambda_{i, l} \operatorname{lk}\left(L_{i}, L_{l}\right) .
$$

Proof. It suffices to work out the degree of $\left[s_{\underline{E}}\right]$, because the generators in one orbit all have the same internal degree as explained in the proof of Theorem 3.9. Let

$$
E=\left\{(i, j) \mid i=1, \ldots, k \text { and } j \in E_{i}\right\}
$$

and set

$$
\bar{E}=\left\{(i, j) \mid i=1, \ldots, k \text { and } j=1, \ldots, n_{i}\right\} \backslash E .
$$

By the discussion of Bar-Natan theory the degree of $\left[s_{\underline{E}}\right]$ is

$$
2 \sum_{\substack{(i, j) \in E \\(l, m) \in \bar{E}}} l k\left(D_{i j}, D_{l m}\right),
$$

where $D_{i j}$ is the $j$-th strand of the $n_{i}$-cable of the $i$-th component of the diagram $D$. Recalling that the strands of each cable are oriented so that strand 1 has the given orientation and that adjacent strands have opposite orientations we see that $l k\left(D_{i j}, D_{l m}\right)=(-1)^{j+m} l k\left(L_{i}, L_{l}\right)$. Thus we have

$$
\begin{aligned}
\operatorname{deg}\left[s_{\underline{E}}\right] & =2 \sum_{\substack{(i, j) \in E \\
(l, m) \in \bar{E}}} l k\left(D_{i j}, D_{l m}\right)=2 \sum_{i=1}^{k} \sum_{l=1}^{k} \sum_{\substack{j \in E_{i} \\
m \in E_{l}}} l k\left(D_{i j}, D_{l m}\right) \\
& =2 \sum_{i=1}^{k} \sum_{l=1}^{k} \sum_{\substack{j \in E_{i} \\
m \in \bar{E}_{l}}}(-1)^{j+m} l k\left(L_{i}, L_{l}\right)=2 \sum_{i=1}^{k} \sum_{l=1}^{k} \lambda_{i, l} l k\left(L_{i}, L_{l}\right) .
\end{aligned}
$$

\section{Khovanov's alternative definition}

There is an alternative way of defining a complex which categorifies the coloured Jones polynomial. This method, suggested by Khovanov, involves reversing the direction of the arrows in the graph of dot-rows. Khovanov conjectures that working over a field of characteristic zero with the Khovanov homology the two complexes give isomorphic homology groups. In this section we prove the analogous statement for the coloured Bar-Natan theory. We note that our proof is valid in Khovanov's original setting too.

Let $\left(C^{*}, \bar{d}\right)$ be the chain complex obtained from the reversed graph. The reversed graph has cables attached to vertices as before and cobordisms attached to 
edges again except this time with the opposite orientation. This way we get a chain complex (rather than a cochain complex) with differential $\bar{d}_{i}: C^{i} \rightarrow C^{i-1}$.

We can identify $\bar{d}$ in terms of $d$. Given any link $L$ there is a nondegenerate inner product on $\mathrm{BN}^{*}(L)$. This inner product is induced by the cylinder cobordism from the link $L \sqcup \bar{L}$ to the empty link. Noting that $\mathrm{BN}^{i}(L) \cong \mathrm{BN}^{i}(\bar{L})$ and that $\mathrm{BN}^{*}(L \sqcup \bar{L}) \cong \mathrm{BN}^{*}(L) \otimes \mathrm{BN}^{*}(\bar{L})$ we see this induces a map $\langle-,-\rangle: \mathrm{BN}^{*}(L) \otimes$ $\mathrm{BN}^{*}(L) \rightarrow \mathbb{F}_{2}$. As is familiar in the discussion of 1+1-dimensional TQFTs the diffeomorphism shown below implies that this inner product is nondegenerate.

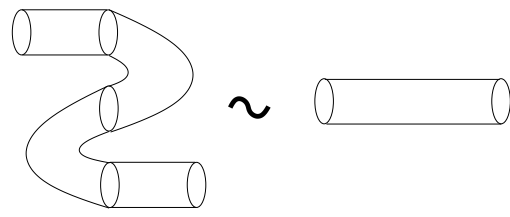

This induces a nondegenerate inner product on the complex $C^{*}$. Let $d_{i}^{*}$ denote the adjoint of $d_{i}$ with respect to this inner product.

Proposition 5.1. $\bar{d}_{i}=d_{i-1}^{*}$.

Proof. In any two-dimensional TQFT the linear map induced by a cylinder commutes with the linear map induced by any cobordism. Therefore the adjoint of the linear map induced by an oriented cobordism is given by the linear map induced by the same cobordism with the opposite orientation.

\section{Theorem 5.2.}

$$
H_{i}\left(C^{*}, \bar{d}\right) \cong H_{i}\left(C^{*}, d\right)
$$

Proof. By linear algebra (analogous to the discussion of harmonic forms in Hodge theory) we see that

$$
H_{i}\left(C^{*}, d\right) \cong \operatorname{Ker} d_{i} \cap \operatorname{Ker} d_{i-1}^{*}
$$

and similarly

$$
H_{i}\left(C^{*}, \bar{d}\right) \cong \operatorname{Ker} \bar{d}_{i} \cap \operatorname{Ker} \bar{d}_{i+1}^{*} .
$$

However using Proposition 5.1 we see that this becomes

$$
H_{i}\left(C^{*}, \bar{d}\right) \cong \operatorname{Ker} d_{i-1}^{*} \cap \operatorname{Ker}\left(d_{i}^{*}\right)^{*}=\operatorname{Ker} d_{i-1}^{*} \cap \operatorname{Ker} d_{i} \cong H_{i}\left(C^{*}, d\right) .
$$

\section{References}

[Bar-Natan 2002] D. Bar-Natan, “On Khovanov's categorification of the Jones polynomial”, Algebr. Geom. Topol. 2 (2002), 337-370. MR 2003h:57014 Zbl 0998.57016

[Bar-Natan 2005] D. Bar-Natan, "Khovanov's homology for tangles and cobordisms", Geom. Topol. 9 (2005), 1443-1499. MR 2006g:57017 Zbl 1084.57011

[Khovanov 2005] M. Khovanov, "Categorifications of the colored Jones polynomial", J. Knot Theory Ramifications 14:1 (2005), 111-130. MR 2006a:57016 Zbl 1083.57019 
[Khovanov 2006] M. Khovanov, "Link homology and Frobenius extensions", Fund. Math. 190 (2006), 179-190. MR MR2232858 Zbl 05039154

[Lee 2005] E. S. Lee, "An endomorphism of the Khovanov invariant", Adv. Math. 197:2 (2005), 554-586. MR 2006g:57024 Zbl 1080.57015

[Rasmussen 2004] J. Rasmussen, "Khovanov homology and the slice genus", preprint, 2004. Available at math.GT/0402131

[Turner 2004] P. Turner, "Calculating Bar-Natan's characteristic two Khovanov homology", preprint, 2004. To appear in J. Knot Theory and its Ramifications. Available at math.GT/0411225

Received June 8, 2005.

MARCO MACKAAY

DePartamento de MATEMÁtica

UNIVERSIDADE DO AlgARVE

CAMPUS DE GAMBELAS

8005-139 FARO

PORTUGAL

mmackaay@ualg.pt

PAUL TURNER

SCHOOL OF MATHEMATICAL AND COMPUTER SCIENCES

HERIOT-WATT UNIVERSITY

EDINBURGH EH14 4AS

SCOTLAND

paul@ma.hw.ac.uk 